\title{
Deterministic Entanglement Swapping in a Superconducting Circuit
}

\author{
Wen Ning ${ }^{1}$, Xin-Jie Huang ${ }^{1}$, Pei-Rong Han ${ }^{1}$, Hekang Li $^{2}$, Hui Deng ${ }^{4,5}$, Zhen-Biao Yang ${ }^{1},{ }^{*}$ \\ Zhi-Rong Zhong ${ }^{1}$, Yan Xia ${ }^{1}$, Kai Xu ${ }^{2,3},{ }^{\dagger}$ Dongning Zheng ${ }^{2,3}$, and Shi-Biao Zheng ${ }^{1 \ddagger}$ \\ 1.Fujian Key Laboratory of Quantum Information and Quantum Optics, \\ College of Physics and Information Engineering, \\ Fuzhou University, Fuzhou, Fujian 350108, China \\ 2.Institute of Physics and Beijing National Laboratory for Condensed Matter Physics, \\ Chinese Academy of Sciences, Beijing 100190, China \\ 3.CAS Center for Excellence in Topological Quantum Computation, \\ University of Chinese Academy of Sciences, Beijing 100190, China \\ 4.Hefei National Laboratory for Physical Sciences at Microscale and Department of Modern Physics, \\ University of Science and Technology of China, Hefei, Anhui 230026, China and \\ 5.CAS Centre for Excellence and Synergetic Innovation Centre in Quantum Information and Quantum Physics, \\ University of Science and Technology of China, Hefei, Anhui 230026, China
}

(Dated: August 13, 2019)

\begin{abstract}
Entanglement swapping, the process to entangle two particles without coupling them in any way, is one of the most striking manifestations of the quantum-mechanical nonlocal characteristic. Besides fundamental interest, this process has applications in complex entanglement manipulation and quantum communication. Here we report a high-fidelity, unconditional entanglement swapping experiment in a superconducting circuit. The measured concurrence characterizing the qubit-qubit entanglement produced by swapping is above 0.75 , confirming most of the entanglement of one qubit with its partner is deterministically transferred to another qubit that has never interacted with it. We further realize delayed-choice entanglement swapping, showing whether two qubits previously behaved as in an entangled state or as in a separable state is determined by a later choice of the type of measurement on their partners. This is the first demonstration of entanglement-separability duality in a deterministic way.

PACS numbers:
\end{abstract}

Quantum entanglement, lying at the heart of the Einstein-Podolsky-Rosen (EPR) paradox [1], is one of the most striking features of quantum mechanics. When two particles are put in an entangled state, they can exhibit nonlocal correlation that cannot be interpreted in terms of any classical model as evidenced by violation of Bell's inequalities [2, 3]. In addition to fundamental tests of quantum mechanics, entanglement is an essential resource for many quantum information tasks, such as quantum teleportation [4] and measurement-based quantum computation [5]. The nonlocal characteristic of quantum-mechanical wave functions allows two particles that have never interacted to be put into an entangled state by means of entanglement swapping [6]. The process is illustrated in Fig. 1, where the two qubits $\left(Q_{1}\right.$ and $\left.Q_{4}\right)$ to be entangled are first entangled with their respective partners $\left(Q_{2}\right.$ and $\left.Q_{3}\right): Q_{1}$ and $Q_{2}$ form the first entangled Bell pair, while $Q_{3}$ and $Q_{4}$ form the second pair. Then a joint Bell state measurement applied to the partners $Q_{2}$ and $Q_{3}$ will project the remaining two qubits, $Q_{1}$ and $Q_{4}$, to one of four possible Bell states; which entangled state is produced depends on the outcome of the Bell state measurement. Aside from fundamental inter-

\footnotetext{
*Electronic address: zbyang@fzu.edu.cn

${ }^{\dagger}$ Electronic address: kaixu@iphy.ac.cn

$\ddagger$ Electronic address: t96034@fzu.edu.cn
}

est, entanglement swapping has practical applications in quantum communication [7] and in multipartite entanglement manipulation necessary for construction of complex quantum networks [8].

Entanglement swapping has been experimentally demonstrated with photonic qubits [9-17]. However, in these optical experiments, entanglement was swapped conditional on the occurrence of preset photon coincidence events. These events were detected only in a small fraction of experimental runs due to the photon loss on optical components, lack of logic operations to completely distinguish all four Bell states, and restriction of photon detectors' efficiency [17]. Experiments have realized heralded entanglement between two spatially separated atomic qubits, each entangled with its emitted photons before a partial Bell state analysis on these photons [18, 19]; the entanglement was swapped also with a small probability. With photonic continuous variables, unconditional entanglement swapping has been reported [20, 21], but where only a small portion of entanglement was preserved after swapping due to the limitation of the degree of entanglement carried by the original entangled beams, which is the main source of the infidelity for teleportation of a continuous-variable state [22]. Although unconditional teleportation has been demonstrated with different kinds of matter qubits, including nuclear magnetic resonance [23], trapped ions $[24,25]$, and superconducting qubits [26, 27], where the teleported states were preset and known to the experi- 


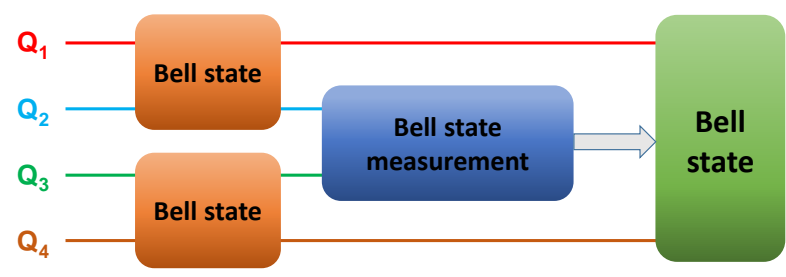

FIG. 1: Sketch of entanglement swapping. Initially, $Q_{1}$ is entangled with $Q_{2}$ in a Bell state, and $Q_{3}$ with $Q_{4}$, but there is no correlation between $Q_{1}$ and $Q_{4}$. A joint measurement on $Q_{2}$ and $Q_{3}$ in the Bell basis will project $Q_{1}$ and $Q_{4}$ to one of four possible Bell states depending on the outcome of the $Q_{2}-Q_{3}$ measurement.

menters, deterministic, high-fidelity entanglement swapping has only been realized in an ion trap [28]. As pointed out in Ref. [10], entanglement swapping is the only known procedure that demonstrates the quantum nature of teleportation-the qubit whose state is to be teleported is entangled with another qubit, rendering it impossible to know this state. When realized in a delayed-choice manner [29], this process reveals a more striking feature, that is, one can a posteriori determine two already detected particles previously behaved as an entangled pair or as a separable pair.

We here implement a deterministic entanglement swapping experiment with superconducting qubits (labeled from $Q_{1}$ to $Q_{4}$ ). Our results show that $Q_{1}$ and $Q_{4}$, although never coupled to each other, are highly entangled after the swapping process, with the measured concurrence above 0.75 . Unlike previous experiments with photonic qubits, the Bell states are produced deterministically and the measurement is single shot, so that the entanglement is swapped unconditionally. We note that an entanglement swapping experiment also with superconducting qubits was briefly mentioned in a recent review [30], but no experimental details have been released up to now. We further realize a delayed-choice entanglement swapping experiment [29], where we choose to perform a Bell state measurement or a separable-state measurement on $Q_{2}$ and $Q_{3}$ after $Q_{1}$ and $Q_{4}$ have been detected. The results demonstrate this later choice decides the previous behavior of $Q_{1}$ and $Q_{4}$-whether they were entangled or separable. This implies that entanglement is not a reality, but is a manifestation of the statistical correlation of the measured data; the same set of data may show different types of correlations and have different interpretations when grouped in different manners.

The device used to perform the experiment swapping is identical to that used in Ref. [31], where a resonator with a fixed frequency $\omega_{r} / 2 \pi=\times 5.588 \mathrm{GHz}$ is controllably coupled to five superconducting Xmon qubits, whose frequencies can be individually adjusted on nanosecond timescales using flux bias lines. The device is sketched in Fig. 2(a), and the optical image shown in Fig. 2(b). Throughout the experiment, $Q_{5}$ (unused) is tuned far off resonance with the resonator and the other qubits, and will not be included in the description of the system. The parameters of the system are detailed in the Supplemental Material [32]. All the qubits and the resonator are initially in their ground states. The experiment starts with applying $\pi$ pulses to $Q_{1}$ and $Q_{3}$, transforming each of them from the ground state $|0\rangle$ to the excited state $|1\rangle$ at its idle frequency, with the experimental sequence shown in Fig. 2(c). Then the qubit pairs $Q_{1}-Q_{2}$ and $Q_{3}-Q_{4}$ are red detuned from the resonator by $\Delta_{1}=\Delta_{2}=2 \pi \times 308$ $\mathrm{MHz}$ and $\Delta_{3}=\Delta_{4}=2 \pi \times 238 \mathrm{MHz}$, respectively. With this setting, the resonator will not exchange photons with the qubits and remain in the ground state, but it can simultaneously mediate two entangling gates, each operating on one qubit pair [35-39], with the coupling between these two qubit pairs being negligible owing to their large detuning [39].

The qubit pair, $Q_{j}-Q_{k}(j=1, k=2$ or $j=3$, $k=4)$, evolves to the Bell state $\left|\Psi_{j, k}^{+}\right\rangle=\left(\left|1_{j}\right\rangle\left|0_{k}\right\rangle+\right.$ $\left.i\left|0_{j}\right\rangle\left|1_{k}\right\rangle\right) / \sqrt{2}$ after the corresponding $\sqrt{\text { iSWAP }}$ gate. As soon as $\left|\Psi_{j, k}^{+}\right\rangle$is generated, $Q_{j}$ and $Q_{k}$ are detuned from each other to stop their coupling. The measured density matrices for these two produced entangled pairs are displayed in the Supplemental Material [32]. Their fidelities to the ideal Bell states are, respectively, $F_{1,2}=0.982 \pm 0.006$ and $F_{3,4}=0.978 \pm 0.007$.

The product of the two Bell states $\left|\Psi_{1,2}^{+}\right\rangle$and $\left|\Psi_{3,4}^{+}\right\rangle$ can be expanded as

$$
\begin{gathered}
|\psi\rangle=\frac{1}{2}\left[-i\left|\Psi_{2,3}^{+}\right\rangle\left|\Psi_{1,4}^{-}\right\rangle+i\left|\Psi_{2,3}^{-}\right\rangle\left|\Psi_{1,4}^{+}\right\rangle\right. \\
\left.+\left|\Phi_{2,3}^{+}\right\rangle\left|\Phi_{1,4}^{+}\right\rangle-\left|\Phi_{2,3}^{-}\right\rangle\left|\Phi_{1,4}^{-}\right\rangle\right]
\end{gathered}
$$

where $\left|\Psi_{j, k}^{ \pm}\right\rangle=\left(\left|1_{j}\right\rangle\left|0_{k}\right\rangle \pm i\left|0_{j}\right\rangle\left|1_{k}\right\rangle\right) / \sqrt{2}$ and $\left|\Phi_{j, k}^{ \pm}\right\rangle=$ $\left(\left|1_{j}\right\rangle\left|1_{k}\right\rangle \pm i\left|0_{j}\right\rangle\left|0_{k}\right\rangle\right) / \sqrt{2}$. To realize entanglement swapping, we perform a measurement on $Q_{2}$ and $Q_{3}$ in the Bell basis $\left\{\left|\Psi_{2,3}^{+}\right\rangle,\left|\Psi_{2,3}^{-}\right\rangle,\left|\Phi_{2,3}^{+}\right\rangle,\left|\Phi_{2,3}^{-}\right\rangle\right\}$, which will project $Q_{1}$ and $Q_{4}$ to one Bell state. A complete Bell state measurement can be implemented by mapping the Bell basis onto the computational basis $\left\{\left|0_{2}\right\rangle\left|0_{3}\right\rangle,\left|0_{2}\right\rangle\left|1_{3}\right\rangle,\left|1_{2}\right\rangle\left|0_{3}\right\rangle,\left|1_{2}\right\rangle\left|1_{3}\right\rangle\right\}$ through a dressedstate phase gate $[40,41]$. We note that the $\sqrt{\text { iSWAP }}$ gate only transforms two out of the four Bell states into product states, and thus cannot be used for deterministically distinguishing all the Bell states. To implement the dressed-state phase gate between $Q_{2}$ and $Q_{3}$, we tune $Q_{1}$ and $Q_{4}$ back to their idle frequencies, so that neither of them can interact with other qubits, and then red detune $Q_{2}$ and $Q_{3}$ from the resonator by the same amount $\Delta_{2}^{\prime}=\Delta_{3}^{\prime}=2 \pi \times 308 \mathrm{MHz}$, switching on their interaction via the resonator-induced virtual photon exchange, with the coupling strength $\lambda_{2,3}=2 \pi \times 1.14 \mathrm{MHz}$. At the same time, we apply a resonant continuous drive to each of these two qubits, whose phase is inverted in the middle of the two-qubit interaction with a duration $\tau_{2,3}=\pi / 2 \lambda_{2,3}$. When the difference of the Rabi frequencies of these two drives is much larger than $\lambda_{2,3}$, a 
(a)

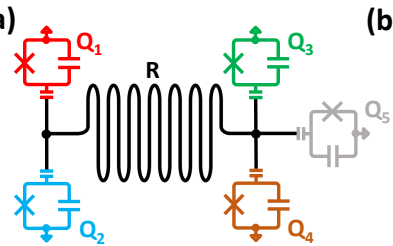

(c)

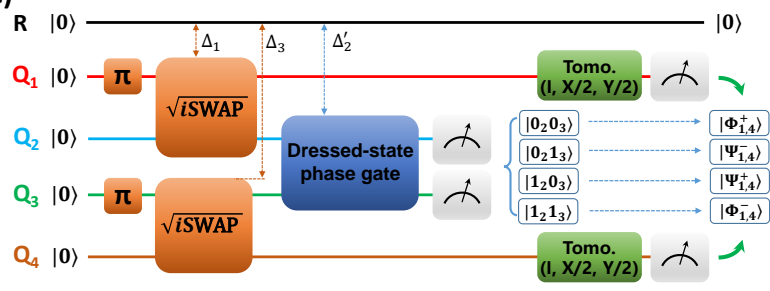

FIG. 2: Device schematic and pulse sequence. (a) Device schematic. Five superconducting Xmon qubits (labeled from $Q_{1}$ to $Q_{5}$ ) are capacitively coupled to a bus resonator $R$. The frequency of each qubit can be adjusted quickly, enabling the relevant qubit-resonator interaction as well as the resonatorinduced qubit-qubit couplings to be effectively switched on and off. (b) Optical image of the device. (c) Experimental sequence. The procedure consists of three parts: Generation of Bell states for qubit pairs $Q_{1}-Q_{2}$ and $Q_{3}-Q_{4}$ via resonatormediated $\sqrt{\text { iSWAP }}$ gates following $\pi$ pulses applied to $Q_{1}$ and $Q_{3} ;$ complete Bell state measurement on $Q_{2}$ and $Q_{3}$, achieved by subsequentially applying a dressed-state phase gate on $Q_{2}$ and $Q_{3}$ and performing a joint detection in the basis $\left\{\left|0_{2}\right\rangle\left|0_{3}\right\rangle,\left|0_{2}\right\rangle\left|1_{3}\right\rangle,\left|1_{2}\right\rangle\left|0_{3}\right\rangle,\left|1_{2}\right\rangle\left|1_{3}\right\rangle\right\} ;$ 2-qubit quantum state tomography for $Q_{1}$ and $Q_{4}$. The detailed pulse sequence is shown in Fig. S3(a) of the Supplemental Material [32].

dressed-state phase gate between $Q_{2}$ and $Q_{3}$ is achieved. As a result, the four Bell states of $Q_{2}$ and $Q_{3}$ evolve as (see Supplemental Material [32])

$$
\begin{aligned}
\left|\Psi_{2,3}^{+}\right\rangle \rightarrow i\left|0_{2}\right\rangle\left|1_{3}\right\rangle,\left|\Psi_{2,3}^{-}\right\rangle \rightarrow\left|1_{2}\right\rangle\left|0_{3}\right\rangle, \\
\left|\Phi_{2,3}^{+}\right\rangle \rightarrow i\left|0_{2}\right\rangle\left|0_{3}\right\rangle,\left|\Phi_{2,3}^{-}\right\rangle \rightarrow\left|1_{2}\right\rangle\left|1_{3}\right\rangle .
\end{aligned}
$$

The combination of this transformation and the subsequent detection of $Q_{2}$ and $Q_{3}$ in the computational basis $\left\{\left|0_{2}\right\rangle\left|0_{3}\right\rangle,\left|0_{2}\right\rangle\left|1_{3}\right\rangle,\left|1_{2}\right\rangle\left|0_{3}\right\rangle,\left|1_{2}\right\rangle\left|1_{3}\right\rangle\right\}$ effectively realizes the complete Bell state analysis, enabling us to distinguish all the four Bell states. Consequently, $Q_{1}$ and $Q_{4}$ are randomly projected onto one of the four Bell states $\left\{\left|\Phi_{1,4}^{+}\right\rangle,\left|\Psi_{1,4}^{-}\right\rangle,\left|\Psi_{1,4}^{+}\right\rangle,\left|\Phi_{1,4}^{-}\right\rangle\right\}$depending on the $Q_{2}-Q_{3}$ measurement outcome. During the $Q_{2}-Q_{3}$ Bellstate measurement, $Q_{1}$ and $Q_{4}$ respectively stay at their idle frequencies, so that the interactions of each of them with the resonator and with any other qubit are effectively switched off due to the large detunings.

After the Bell state analysis, we perform joint 2-qubit state tomography to reconstruct the density matrix for $Q_{1}$ and $Q_{4}$. The measured density matrices of $Q_{1}$ and $Q_{4}$ conditional on the measurement outcomes $\left|0_{2}\right\rangle\left|0_{3}\right\rangle$, $\left|0_{2}\right\rangle\left|1_{3}\right\rangle,\left|1_{2}\right\rangle\left|0_{3}\right\rangle$, and $\left|1_{2}\right\rangle\left|1_{3}\right\rangle$ of $Q_{2}$ and $Q_{3}$ are displayed in Figs. 3(a)-(d), respectively. The readout error of each qubit is corrected when reconstructing these density matrices. Ideally, for these four outcomes $Q_{1}$
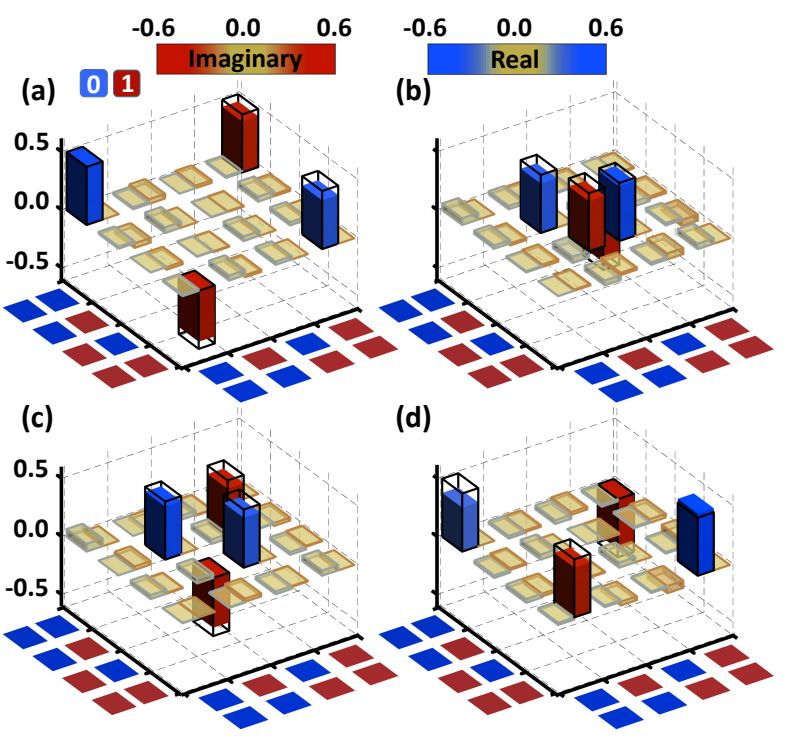

FIG. 3: Measured $Q_{1}-Q_{4}$ density matrices conditional on the four $Q_{2}-Q_{3}$ measurement outcomes: (a) $\left|0_{2}\right\rangle\left|0_{3}\right\rangle$; (b) $\left|0_{2}\right\rangle\left|1_{3}\right\rangle$; (c) $\left|1_{2}\right\rangle\left|0_{3}\right\rangle$; (d) $\left|1_{2}\right\rangle\left|1_{3}\right\rangle$. The results are obtained with the experiment sequence shown in Fig. 2(c). Each matrix element is characterized by two color hbars, one for the real part and the other for the imaginary part. The black wire frames denote the matrix elements of the ideal output states.

and $Q_{4}$ are projected onto $\left|\Phi_{1,4}^{+}\right\rangle,\left|\Psi_{1,4}^{-}\right\rangle,\left|\Psi_{1,4}^{+}\right\rangle$, and $\left|\Phi_{1,4}^{-}\right\rangle$, respectively. The fidelities for the four obtained Bell states to the ideal ones are $F_{\Phi^{+}}=0.893 \pm 0.010$, $F_{\Psi^{-}}=0.879 \pm 0.010, F_{\Psi^{+}}=0.872 \pm 0.011$, and $F_{\Phi^{-}}=$ $0.884 \pm 0.010$, with the concurrences $C_{\Phi^{+}}=0.794 \pm 0.020$, $C_{\Psi^{-}}=0.779 \pm 0.020, C_{\Psi^{+}}=0.758 \pm 0.024$, and $C_{\Phi^{-}}=$ $0.785 \pm 0.021$, respectively. These results show that $Q_{4}$ $\left(Q_{1}\right)$ inherits most of the entanglement of $Q_{2}$ with $Q_{1}$ $\left(Q_{3}\right.$ with $\left.Q_{4}\right)$ after the swapping, which is in stark contrast with experiments with photonic continuous variables [20,21], where only a small portion of entanglement is inherited (e.g., about $29 \%$ in Ref. [20]). As the readout error of each qubit is corrected when reconstructing the $Q_{1}-Q_{4}$ output density matrices, the infidelities mainly come from imperfect preparation of the $Q_{1}-Q_{2}$ and $Q_{3^{-}}$ $Q_{4}$ Bell states, imperfection of the $Q_{2}-Q_{3}$ dressed-state phase gate, and decoherence effects of $Q_{1}$ and $Q_{4}$ during this gate.

We note that the deterministic entanglement swapping requires reliable Bell state measurement on $Q_{2}$ and $Q_{3}$, whose performance depends on the quality of the dressed-state phase gate and the single-shot state readout fidelities of $Q_{2}$ and $Q_{3}$. The fidelity of the dressedstate phase gate is $F_{g t} \approx 0.966$, while the average readout fidelities of $Q_{2}$ and $Q_{3}$ are $F_{2}=0.95$ and $F_{3}=0.94$, where $F_{j}=\left(F_{0, j}+F_{1, j}\right) / 2$, with $F_{0, j}$ and $F_{1, j}$ denoting the $|0\rangle$ - and $|1\rangle$-state readout fidelities of $Q_{j}$, whose values are listed in Table S1 of the Supplemental Material [32]. Without readout error corrections, the average 


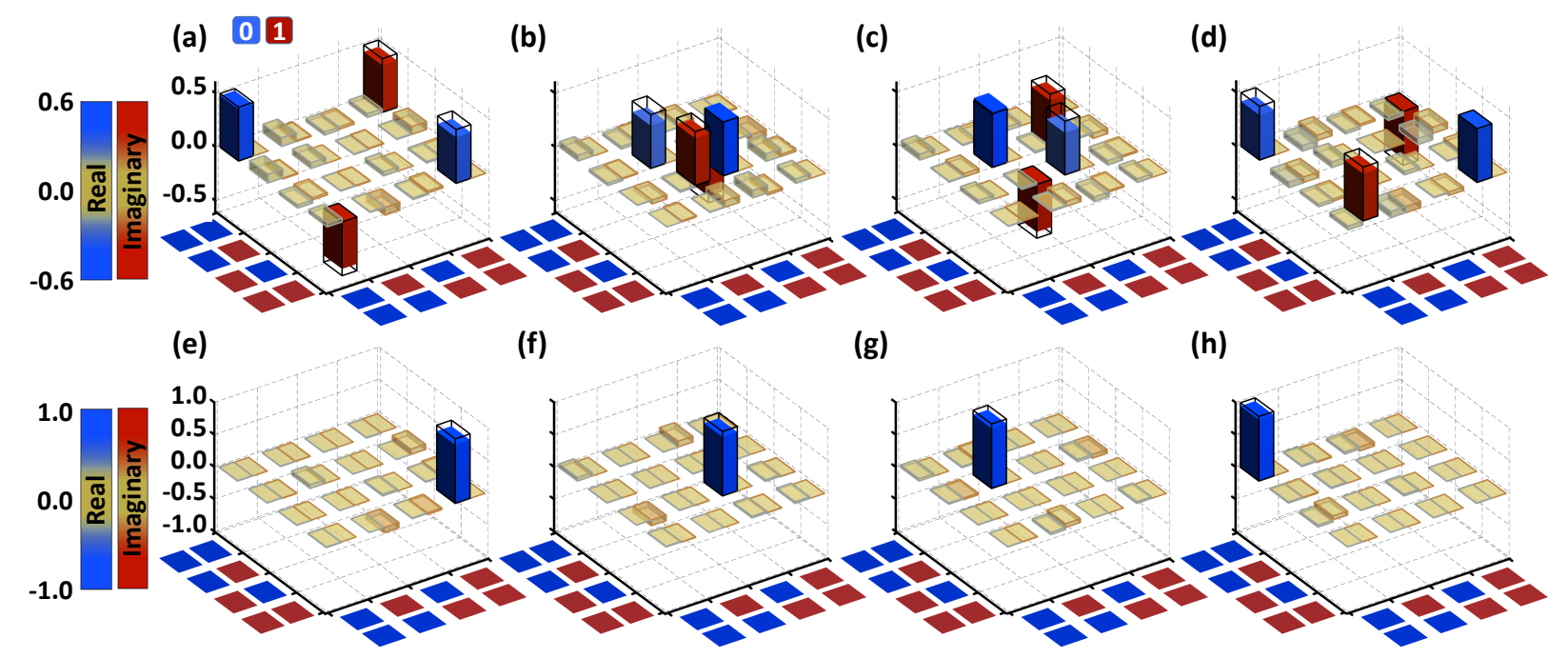

FIG. 4: Measured $Q_{1}-Q_{4}$ density matrices conditional on outcomes of delayed-choice $Q_{2}-Q_{3}$ measurement. (a)-(d) Results obtained from the four subsets of data correlated with the outcomes $\left\{\left|0_{2}\right\rangle\left|0_{3}\right\rangle,\left|0_{2}\right\rangle\left|1_{3}\right\rangle,\left|1_{2}\right\rangle\left|0_{3}\right\rangle,\left|1_{2}\right\rangle\left|1_{3}\right\rangle\right\}$ of $Q_{2}$ - $Q_{3}$ measurement performed after the dressed-state phase gate. Compared with Fig. 2(c), the temporal orders of $Q_{2}-Q_{3}$ Bell measurement and $Q_{1}-Q_{4}$ joint state tomography are inverted, with the experimental pulse sequence shown in the Supplemental Material [32]. (e)-(h) Results obtained from the four subsets of data correlated with the outcomes of the later $Q_{2}$ - $Q_{3}$ measurement without the dressed-state phase gate.

measurement fidelity of the four Bell states is roughly $F_{g t} F_{2} F_{3} \approx 0.863$, and each of the four corresponding $Q_{1}-Q_{4}$ output states, as shown in Fig. S4 of the Supplemental Material [32], has a fidelity above 0.76 and a concurrence exceeding 0.54 .

Going one step further, we delay the $Q_{2}-Q_{3}$ Bell state measurement until the joint $Q_{1}-Q_{4}$ state has been detected. The detailed pulse sequence is shown in Fig. S3(b) of the Supplemental Material [32], where the $Q_{2^{-}}$ $Q_{3}$ readout pulse is applied about 219 ns after the end of $Q_{1}-Q_{4}$ readout pulse. Since the correlation between the outcomes of $Q_{2}-Q_{3}$ measurement and $Q_{1}-Q_{4}$ measurement is independent of their temporal order, this arrangement will result in entanglement swapping in a delayed manner [29]. According to $Q_{2}-Q_{3}$ Bell state measurement outcomes, the data of $Q_{1}-Q_{4}$ joint state measurement are sorted into four subsets, from which four density matrices are reconstructed, and shown in Figs. 4(a)-(d). As in the nondelayed case, these four density matrices correspond to four Bell states, with the respective fidelities $F_{\Phi^{+}}=0.891 \pm 0.012, F_{\Psi^{-}}=0.891 \pm$ $0.012, F_{\Psi^{+}}=0.896 \pm 0.010$, and $F_{\Phi^{-}}=0.897 \pm 0.010$, and concurrences $C_{\Phi^{+}}=0.815 \pm 0.026, C_{\Psi^{-}}=0.816 \pm 0.024$, $C_{\Psi^{+}}=0.806 \pm 0.022$, and $C_{\Phi^{-}}=0.807 \pm 0.019$. The fidelities and concurrences are slightly higher than those in the nondelayed case due to the fact that $Q_{1}-Q_{4}$ joint state is detected earlier so that the measured data are less affected by decoherence effects.

We also perform another experiment, where we choose to measure $Q_{2}$ and $Q_{3}$ in the computational basis (without performing the dressed-state phase gate before detection of their states). Again, this measurement is performed after $Q_{1}-Q_{4}$ joint state detection, with the pulse sequence shown in Fig. S3(c) of the Supplemental Material [32]. The density matrices reconstructed from the four subsets of $Q_{1}-Q_{4}$ measurement data, each associated with one of $Q_{2}-Q_{3}$ measurement outcomes $\left\{\left|0_{2}\right\rangle\left|0_{3}\right\rangle,\left|0_{2}\right\rangle\left|1_{3}\right\rangle,\left|1_{2}\right\rangle\left|0_{3}\right\rangle,\left|1_{2}\right\rangle\left|1_{3}\right\rangle\right\}$, are presented in Figs. 4(e)-(h), respectively. As expected, these matrices correspond to product states $\left\{\left|1_{1}\right\rangle\left|1_{4}\right\rangle,\left|1_{1}\right\rangle\left|0_{4}\right\rangle,\left|0_{1}\right\rangle\left|1_{4}\right\rangle,\left|0_{1}\right\rangle\left|0_{4}\right\rangle\right\}$ with the fidelities $\{0.907 \pm 0.011,0.914 \pm 0.009,0.930 \pm 0.009,0.949 \pm 0.008\}$. The concurrence associated with each of these reconstructed matrices is approximate to 0 (see Table S4 of the Supplemental Material [32]).

The above results demonstrate whether or not the already measured qubits $Q_{1}$ and $Q_{4}$ previously behaved as an entangled pair depends on the later choice of the type of measurement on $Q_{2}$ and $Q_{3}$. As a generalization of Wheeler's delayed-choice experiment proposed for illustrating the wave-particle duality of a single particle [42], the delayed-choice entanglement swapping experiment reveals the entanglement-separability duality of two particles [43]. A realization of this gedanken experiment was previously reported with photonic qubits [17], but where only two out of four basis states could be distinguished in each of the two mutually exclusive measurements, so that the entanglement-separability duality was only partially demonstrated: Whether the $Q_{1}-Q_{4}$ states associated with the two indistinguishable $Q_{2}-Q_{3}$ basis states manifested a quantum or a classical correlation could not be confirmed. Another problem is only a small fraction of events coinciding with the distinguishable basis states was detected owing to the photon loss on optical components (only $4.4 \%$ photons left) and nonunity photon detection efficiency. 
We have demonstrated deterministic entanglement swapping with superconducting qubits controllably coupled to a resonator. The qubit-qubit couplings mediated by the resonator allows for both the controlled generation of the Bell states and complete Bell state analysis. We have further deterministically realized delayed-choice entanglement swapping, demonstrating whether two qubits exhibited entangled or separable behavior can be a posteriori decided after they have been measured. Our results indicate quantum entanglement of two quantum systems is a manifestation of the statistical correlations of the measured data, instead of a reality.

We thank Haohua Wang at Zhejiang University for technical support. This work was supported by the National Natural Science Foundation of China (Grants No. 11674060, No. 11874114, and No. 11875108), and the Strategic Priority Research Program of Chinese Academy of Sciences (Grant No. XDB28000000).
[1] A. Einstein, B. Podolsky, and N. Rosen, Phys. Rev. 47, 777 (1935).

[2] J. S. Bell, Physics 1, 195 (1965).

[3] J. F. Clauser, M. A. Horne, A. Shimony, and R. A. Holt, Phys. Rev. Lett. 23, 880 (1969).

[4] C. H. Bennett, G. Brassard, C. Crépeau, R. Jozsa, A. Peres, and W. K. Wootters, Phys. Rev. Lett. 70, 1895 (1993).

[5] R. Raussendorf and H. J. Briegel, Phys. Rev. Lett. 86, 5188 (2001).

[6] M. Żukowski, A. Zeilinger, M. A. Horne, and A. K. Ekert, Phys. Rev. Lett. 71, 4287 (1993).

[7] W. Dür, H.-J. Briegel, J. I. Cirac, and P. Zoller, Phys. Rev. A 59, 169 (1999).

[8] S. Bose, V. Vedral, and P. L. Knight, Phys. Rev. A 57, 822 (1998).

[9] J.-W. Pan, D. Bouwmeester, H. Weinfurter, and A. Zeilinger, Phys. Rev. Lett. 80, 3891 (1998).

[10] J.-W. Pan, M. Daniell, S. Gasparoni, G. Weihs, and A. Zeilinger, Phys. Rev. Lett. 86, 4435 (2001).

[11] H. de Riedmatten, I. Marcikic, J. A. W. van Houwelingen, W. Tittel, H. Zbinden, and N. Gisin, Phys. Rev. A 71, 050302(R) (2005).

[12] M. Halder, A. Beveratos, N. Gisin, V. Scarani, C. Simon, and H. Zbinden, Nat. Phys. 3, 692 (2007).

[13] C.-Y. Lu, T. Yang, and J.-W. Pan, Phys. Rev. Lett. 103, 020501 (2009).

[14] C. Schmid, N. Kiesel, U. K. Weber, R. Ursin, A. Zeilinger, and H. Weinfurter, New J. Phys. 11, 033008 (2009).

[15] F. Sciarrino, E. Lombardi, G. Milani, and F. De Martini, Phys. Rev. A 66, 024309 (2002).

[16] T. Jennewein, G. Weihs, J.-W. Pan, and A. Zeilinger, Phys. Rev. Lett. 88, 017903 (2001).

[17] X.-S. Ma, S. Zotter, J. Kofler, R. Ursin, T. Jennewein, C. Brukner, and A. Zeilinger, Nat. Phys. 8, 479 (2012).

[18] D. N. Matsukevich, P. Maunz, D. L. Moehring, S. Olmschenk, and C. Monroe, Phys. Rev. Lett. 100, 150404 (2008).

[19] Z.-S. Yuan, Y.-A. Chen, B. Zhao, S. Chen, J. Schmiedmayer, and J.-W. Pan, Nature (London) 454, 1098 (2008).

[20] X. Jia, X. Su, Q. Pan, J. Gao, C. Xie, and K. Peng, Phys. Rev. Lett. 93, 250503 (2004).

[21] N. Takei, H. Yonezawa, T. Aoki, and A. Furusawa, Phys. Rev. Lett. 94, 220502 (2005).

[22] S. L. Braunstein, and H. J. Kimble, Phys. Rev. Lett. 80, 869 (1998).

[23] M. A. Nielsen, E. Knill, and R. Laflamme, Nature (London) 396, 52 (1998).
[24] M. Riebe, H. Häffner, C. F. Roos, W. Hänsel, J. Benhelm, G. P. T. Lancaster, T. W. Körber, C. Becher, F. SchmidtKaler, D. F. V. James, and R. Blatt, Nature (London) 429, 734 (2004).

[25] M. D. Barrett, J. Chiaverini, T. Schaetz, J. Britton, W. M. Itano, J. D. Jost, E. Knill, C. Langer, D. Leibfried, R. Ozeri, and D. J. Wineland, Nature (London) 429, 737 (2004).

[26] M. Baur, A. Fedorov, L. Steffen, S. Filipp, M. P. da Silva, and A. Wallraff, Phys. Rev. Lett. 108, 040502 (2012).

[27] L. Steffen, A. Fedorov, M. Oppliger, Y. Salathe, P. Kurpiers, M. Baur, G. Puebla-Hellmann, C. Eichler, and A. Wallraff, Nature (London) 500, 319 (2013).

[28] M. Riebe, T. Monz, K. Kim, A. S. Villar, P. Schindler, M. Chwalla, M. Hennrich, and R. Blatt, Nat. Phys. 4, 839 (2008).

[29] A. Peres, J. Mod. Opt. 47, 139 (2000).

[30] G. Wendin, Rep. Prog. Phys. 80, 106001 (2017).

[31] C. Song, S.-B. Zheng, P. Zhang, K. Xu, L. Zhang, Q. Guo, W. Liu, D. Xu, H. Deng, K. Huang, D. Zheng, X. Zhu, and H. Wang, Nat. Commun. 8, 1061 (2017).

[32] See Supplemental Material at $<$ url $>$, which includes Refs. [33, 34], for the device parameters, characterization of the Bell states produced by $\sqrt{\text { iSWAP }}$ gates, realization of complete Bell state measurement, detailed experimental pulse sequences, results without readout error corrections for Bell state measurement, numerical simulations, experimental setup, and details on qubits' readout.

[33] K. Xu, J.-J. Chen, Y. Zeng, Y.-R. Zhang, C. Song, W. Liu, Q. Guo, P. Zhang, D. Xu, H. Deng, K. Huang, H. Wang, X. Zhu, D. Zheng, and H. Fan, Phys. Rev. Lett. 120, 050507 (2018).

[34] D. Sank, Ph. D. thesis, University of California Santa Barbara, 2014.

[35] S.-B. Zheng and G.-C. Guo, Phys. Rev. Lett. 85, 2392 (2000).

[36] S. Osnaghi, P. Bertet, A. Auffeves, P. Maioli, M. Brune, J. M. Raimond, and S. Haroche, Phys. Rev. Lett. 87, 037902 (2001).

[37] J. Majer, J. M. Chow, J. M. Gambetta, Jens Koch, B. R. Johnson, J. A. Schreier, L. Frunzio, D. I. Schuster, A. A. Houck, A. Wallraff, A. Blais, M. H. Devoret, S. M. Girvin, and R. J. Schoelkopf, Nature (London) 449, 443 (2007).

[38] Y.-P. Zhong, D. Xu, P. Wang, C. Song, Q.-J. Guo, W.-X. Liu, K. Xu, B.-X. Xia, C.-Y. Lu, Siyuan Han, J.-W. Pan, and H. Wang, Phys. Rev. Lett. 117, 110501 (2016).

[39] C. Song, K. Xu, W. Liu, C.-P. Yang, S.-B. Zheng, H. Deng, Q. Xie, K. Huang, Q. Guo, L. Zhang, P. Zhang, D. Xu, D. Zheng, X. Zhu, H. Wang, Y.-A. Chen, C.-Y. 
Lu, S. Han, and J.-W. Pan, Phys. Rev. Lett. 119, 180511 (2017).

[40] Q. Guo, S.-B. Zheng, J. Wang, C. Song, P. Zhang, K. Li, W. Liu, H. Deng, K. Huang, D. Zheng, X. Zhu, H. Wang, C.-Y. Lu, and J.-W. Pan, Phys. Rev. Lett. 121, 130501 (2018).

[41] C. Song, D. Xu, P. Zhang, J. Wang, Q. Guo, W. Liu, K. Xu, H. Deng, K. Huang, D. Zheng, S.-B. Zheng, H.
Wang, X. Zhu, C.-Y. Lu, and J.-W. Pan, Phys. Rev. Lett. 121, 030502 (2018).

[42] J. A. Wheeler, in Quantum Theory and Measurement, edited by J. A. Wheeler and W. H. Zurek (University Press, Princeton, NJ, 1984), p. 182.

[43] Č. Brukner, M. Aspelmeyer, and A. Zeilinger, Found. Phys. 35, 1909 (2005). 


\title{
Supplemental Material for "Deterministic entanglement swapping in a superconducting circuit"
}

\author{
Wen Ning ${ }^{1}$, Xin-Jie Huang ${ }^{1}$, Pei-Rong $\operatorname{Han}^{1}$, Hekang Li $^{2}$, Hui Deng ${ }^{4,5}$, Zhen-Biao Yang ${ }^{1},{ }^{*}$

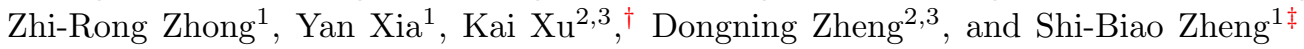 \\ 1.Fujian Key Laboratory of Quantum Information and Quantum Optics, \\ College of Physics and Information Engineering, \\ Fuzhou University, Fuzhou, Fujian 350108, China \\ 2.Institute of Physics and Beijing National Laboratory for Condensed Matter Physics, \\ Chinese Academy of Sciences, Beijing 100190,China \\ 3.CAS Center for Excellence in Topological Quantum Computation, \\ University of Chinese Academy of Sciences, Beijing 100190, China \\ 4.Hefei National Laboratory for Physical Sciences at Microscale and Department of Modern Physics, \\ University of Science and Technology of China, Hefei, Anhui 230026, China and \\ 5.CAS Centre for Excellence and Synergetic Innovation Centre in Quantum Information and Quantum Physics, \\ University of Science and Technology of China, Hefei, Anhui 230026, China
}

(Dated: August 13, 2019)

PACS numbers:

\section{Contents}

1. Device parameters

2. Characterization of Bell states produced by $\sqrt{i \mathrm{SWAP}}$ gates

3. Complete Bell state measurement

4. Experimental pulse sequences

5. Results without readout error corrections for Bell state measurement

6. Numerical results

7. Experimental setup

8. Qubit readout

References

\section{DEVICE PARAMETERS}

The device used to perform the present experiment is the same as that reported in Ref. [1], where five frequency-tunable superconducting Xmon qubits, labeled from $Q_{1}$ to $Q_{5}$, are capacitively coupled to a common resonator. In our experiment, $Q_{5}$ is not used. The full Hamiltonian of the system can be described as

\footnotetext{
*Electronic address: zbyang@fzu.edu.cn

$\dagger$ Electronic address: kaixu@iphy.ac.cn

${ }^{\ddagger}$ Electronic address: t96034@fzu.edu.cn
}

$1 \quad H=\hbar \omega_{r} a^{+} a+\hbar \sum_{j=1}^{5} \omega_{j} S_{j}^{+} S_{j}^{-}+\hbar \sum_{j=1}^{5} g_{j}\left(S_{j}^{+} a+S_{j}^{-} a^{+}\right)$

$$
+\hbar \sum_{j, k} \lambda_{j, k}^{c}\left(S_{j}^{+} S_{k}^{-}+S_{k}^{+} S_{j}^{-}\right)
$$

Qubit frequencies $\omega_{j} / 2 \pi$ are individually tunable from 5 to $6 \mathrm{GHz}$ while the resonator frequency $\omega_{r} / 2 \pi$ is fixed at about $5.588 \mathrm{GHz} . g_{j}$ is the coupling strength between qubit $Q_{j}$ and the resonator with the magnitude listed in Table S1. By equally detuning the frequency of any two qubits far away from that of the resonator, we can realize the effective qubit-qubit interaction with the coupling strength of $g_{j} g_{k} / \Delta\left(\Delta=\omega_{j}-\omega_{r}=\omega_{k}-\omega_{r},|\Delta| \gg g_{j}, g_{k}\right)$, which enables realizations of the $\sqrt{i \text { SWAP }}$ gate and dressed-state phase gate used in the experiment. Note that except for the dominant resonator-mediated interaction, there exists very small direct couplings $\lambda_{j, k}^{c}$ in the system which have been reported elsewhere in similar devices $[2,3]$.

Characterization of qubit performance is presented in Table S1. For technical details about the superconducting qubits, see Supplemental Material of Ref. [2], which shares similar control methods to our experiment.

\section{CHARACTERIZATION OF BELL STATES PRODUCED BY $\sqrt{i \text { SWAP }}$ GATES}

The original $Q_{1}-Q_{2}$ and $Q_{3}-Q_{4}$ Bell states are produced simultaneously [2]. The parallel operations for generating these entangled states are realized by tuning the frequencies of these two qubit pairs to $5.28 \mathrm{GHz}$ and $5.35 \mathrm{GHz}$, respectively. This frequency setting enables the resonator to mediate two independent qubit-qubit swapping interactions, one between $Q_{1}$ and $Q_{2}$ and the 


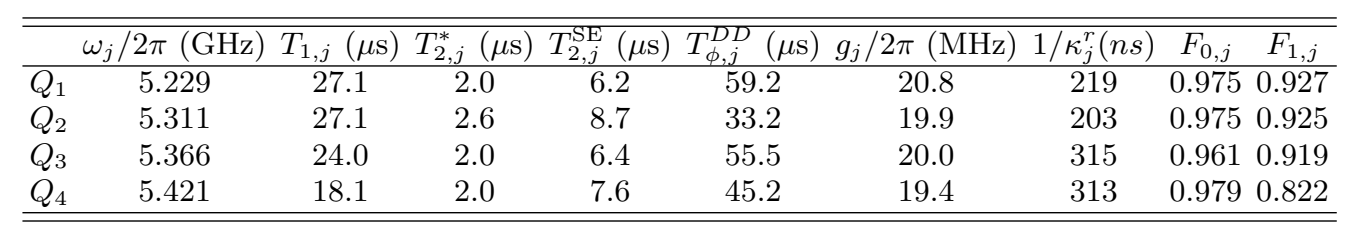

TABLE S1: Qubits characteristics. $\omega_{j} / 2 \pi$ is the idle frequency of $Q_{j}$ where single-qubit rotation pulses and tomographic pulses are applied. $T_{1, j}$ and $T_{2, j}^{*}$ are the energy relaxation time and Ramsey dephasing time of $Q_{j}$ (Gaussian decay) respectively measured at the idle point. $T_{2, j}^{\mathrm{SE}}$ is the dephasing time (Gaussian decay) with spin echo, while $T_{\phi, j}^{D D}$ denotes the dephasing time (exponential decay) under continuous driving for dynamical decoupling [3]. The continuous driving is used in our experiment as it protects the qubits from dephasing much more effectively compared with the spin-echo technique. $g_{j}$ is the coupling strength between $Q_{j}$ and the bus resonator, $\kappa_{j}^{r}$ represents the linewidth of the readout resonator for measuring the state of $Q_{j}$. $F_{0, j}$ $\left(F_{1, j}\right)$ is the probability of detecting $Q_{j}$ in $|0\rangle(|1\rangle)$ when it is prepared in $|0\rangle(|1\rangle)$ state.

other between $Q_{3}$ and $Q_{4}$. We note that the magnitude of the measured coupling $\lambda_{j, k}$ between qubits $Q_{j}$ and $Q_{k}$ is slightly smaller than the calculated resonatorinduced coupling. This is due to the fact that direct coupling between these qubits with an opposite sign partly cancels out the resonator-induced coupling $[2,3]$. We characterize the Bell states generated via the corresponding $\sqrt{i \mathrm{SWAP}}$ gates through joint state tomography, with the $Q_{1}-Q_{2}$ and $Q_{3}-Q_{4}$ density matrices displayed in Fig. S1(a) and (b), respectively. The entanglements in the Bell states are characterized by the magnitudes of the off-diagonal matrix elements $\rho_{01,10}$ and $\rho_{10,01}$, which are about 0.49 for both produced Bell pairs. The fidelities of these produced Bell states are respectively $F_{1,2}=0.982 \pm 0.006$ and $F_{3,4}=0.978 \pm 0.007$. The populations of $\left|0_{1}\right\rangle\left|1_{2}\right\rangle\left|0_{3}\right\rangle\left|1_{4}\right\rangle,\left|0_{1}\right\rangle\left|1_{2}\right\rangle\left|1_{3}\right\rangle\left|0_{4}\right\rangle$, $\left|1_{1}\right\rangle\left|0_{2}\right\rangle\left|0_{3}\right\rangle\left|1_{4}\right\rangle$, and $\left|1_{1}\right\rangle\left|0_{2}\right\rangle\left|1_{3}\right\rangle\left|0_{4}\right\rangle$ in the joint computational basis of the four qubits are $0.248 \pm 0.003$, $0.241 \pm 0.006, \quad 0.247 \pm 0.003$, and $0.240 \pm 0.004$, respectively. Slight deviations of these populations from $1 / 4$ are due to decoherence effects of these qubits and limited detunings between each pair of qubits and the resonator and between these two qubit pairs.

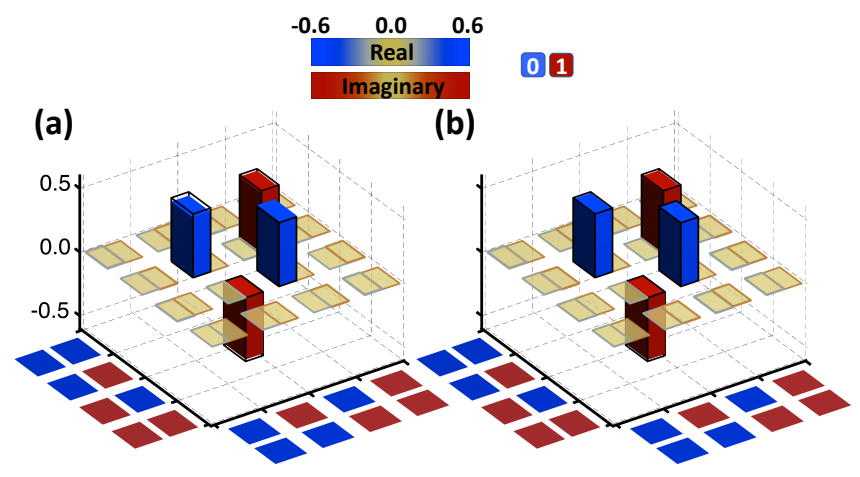

FIG. S1: Measured density matrices of Bell states produced via $\sqrt{i \text { SWAP }}$ gates. (a) $Q_{1}-Q_{2}$ density matrix. (b) $Q_{3}-Q_{4}$ density matrix. Each qubit pair is prepared in the state $|1\rangle|0\rangle$ before the corresponding $\sqrt{i \text { SWAP }}$ gate. The real and imaginary parts of each matrix element are separately characterized with two different color bars. The black wire frames represent the matrix elements of the ideal Bell states.
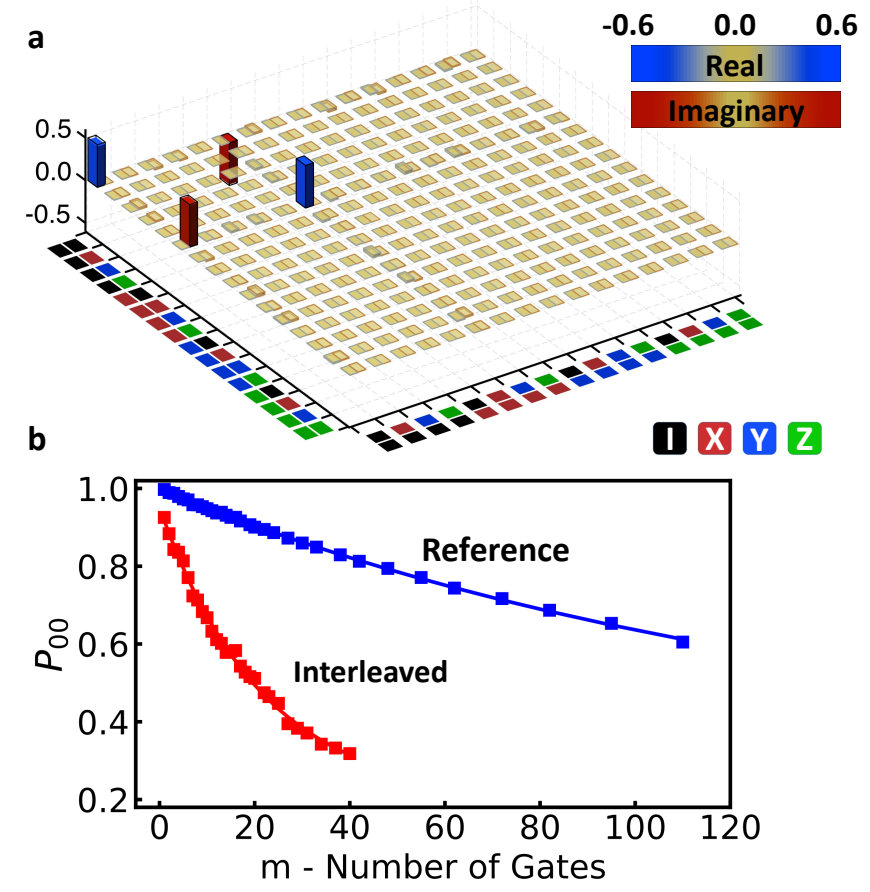

FIG. S2: Characterization of dressed-state phase gate. (a) Measured $\chi$ matrix for the dressed-state phase gate in the Pauli basis. The real and imaginary parts of each element are plotted separately with different colorbars. The black wire frames represent the elements of ideal $\chi$. (b) Randomized benchmarking by inserting the dressed-state phase gate between random single-qubit Pauli gates. Plotted are the corrected probability of $|00\rangle$ state after a series of random Pauli gates with (red) and without (blue) the dressed-state phase gate inserted.

\section{COMPLETE BELL STATE MEASUREMENT}

In our experiment, the $Q_{2}-Q_{3}$ Bell state measurement is enabled by a dressed-state phase gate realized by a combination of the resonator-mediated $Q_{2}-Q_{3}$ swapping coupling and continuous resonant driving. When $Q_{2}$ and $Q_{3}$ are red detuned from the resonator by the same amount $\Delta_{2}^{\prime}=\Delta_{3}^{\prime}$ that is much larger than the corresponding qubit-resonator couplings, the resonatorinduced $Q_{2}-Q_{3}$ coupling strength is $\lambda_{2,3}=g_{2} g_{3} / \Delta_{2}^{\prime}$ [2]. Under continuous driving, the dynamics of $Q_{2}$ and $Q_{3}$ is 
described by the effective Hamiltonian [3]

$$
H_{\mathrm{eff}}=\hbar\left(-\lambda_{2,3} S_{2}^{+} S_{3}^{-}+\sum_{j=2,3} \Omega_{j} e^{i \varphi_{j}} S_{j}^{+}\right)+H . c .
$$

where $\Omega_{j}$ and $\varphi_{j}$ denote the Rabi frequency and phase of the drive applied to $Q_{j}$. We here assume that $\varphi_{2}=\varphi_{3}=$ $\varphi$. Under the condition $\left|\Omega_{2}-\Omega_{3}\right| \gg\left|\lambda_{2,3}\right|$, the effective Hamiltonian approximates

$$
H_{\mathrm{eff}}^{\prime}=-\frac{1}{2} \hbar \lambda_{2,3} S_{z, \varphi, 2} S_{z, \varphi, 3}+\hbar \sum_{j=2,3} \Omega_{j} S_{z, \varphi, j}
$$

where $S_{z, \varphi, j}=\left|+_{\varphi, j}\right\rangle\left\langle+_{\varphi, j}|-|-_{\varphi, j}\right\rangle\left\langle-{ }_{\varphi, j}\right|$, with $\left|+_{\varphi, j}\right\rangle$ and $\left|-{ }_{\varphi, j}\right\rangle$ being the dressed states, defined as $\left|+{ }_{\varphi, j}\right\rangle=\left(\left|0_{j}\right\rangle+e^{i \varphi}\left|1_{j}\right\rangle\right) / \sqrt{2}$ and $\left|-{ }_{\varphi, j}\right\rangle=\left(\left|0_{j}\right\rangle-e^{i \varphi}\left|1_{j}\right\rangle\right) / \sqrt{2}$. When the phase of each drive is inverted in the middle of the pulse with the duration $\tau=\pi / 2 \lambda_{2,3}$, this effective Hamiltonian leads to the controlled $\pi$-phase gate in the dressed-state basis $\left\{\left|+_{\varphi, 2}\right\rangle\left|+_{\varphi, 3}\right\rangle,\left|+_{\varphi, 2}\right\rangle\left|-_{\varphi, 3}\right\rangle,\left|-{ }_{\varphi, 2}\right\rangle\left|+_{\varphi, 3}\right\rangle,\left|-{ }_{\varphi, 2}\right\rangle\left|-{ }_{\varphi, 3}\right\rangle\right\}$ up to single-qubit operations $\exp \left(i \pi S_{z, \varphi, j} / 4\right)$. For simplicity, we take $\varphi=0$. Then the evolution operator in the computational basis $\left\{\left|0_{2}\right\rangle\left|0_{3}\right\rangle,\left|0_{2}\right\rangle\left|1_{3}\right\rangle,\left|1_{2}\right\rangle\left|0_{3}\right\rangle,\left|1_{2}\right\rangle\left|1_{3}\right\rangle\right\}$ is

$$
U=\frac{1}{\sqrt{2}}\left(\begin{array}{cccc}
1 & 0 & 0 & i \\
0 & 1 & i & 0 \\
0 & i & 1 & 0 \\
i & 0 & 0 & 1
\end{array}\right)
$$

After the application of this evolution operator, each of the four Bell states is transformed to a computational state, with the correspondence given by Eq. (3) of the main text.

The dressed-state phase gate is characterized by both quantum process tomography and randomized benchmarking in experiment. The $\chi$ matrix obtained by quantum process tomography is shown in Fig. S2, with a fidelity of $0.966 \pm 0.005$, in agreement with that characterized by randomized benchmarking, which yields a fidelity of $0.971 \pm 0.002$.

\section{EXPERIMENTAL PULSE SEQUENCES}

The pulse sequence for entanglement swapping in the normal temporal order is shown in Fig. S3(a), where the time interval between $Q_{2}-Q_{3}$ readout pulse and $Q_{1}-Q_{4}$ readout pulse is about 40 ns. In our delayed-choice entanglement swapping experiment, we choose to measure $Q_{2}$ and $Q_{3}$ in the Bell basis or in the computational basis after joint $Q_{1}-Q_{4}$ measurement has been performed. The pulse sequence with the choice of measurement in the Bell basis is shown in Fig. S3(b), where $Q_{2}-Q_{3}$ readout pulse is applied about $219 \mathrm{~ns}$ after the end of $Q_{1}-Q_{4}$ readout pulse. With this pulse sequence, the density matrices for
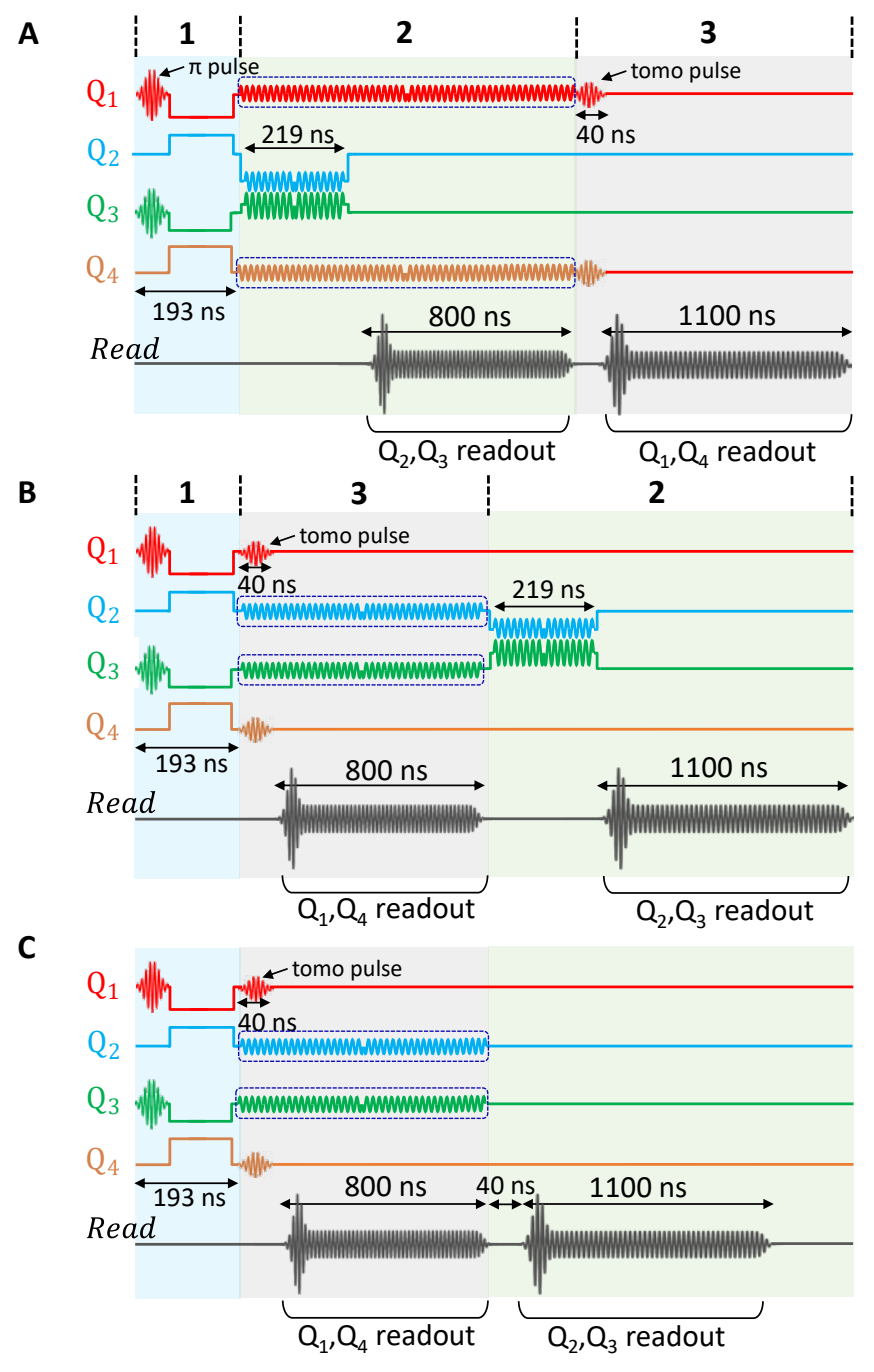

FIG. S3: Experimental pulse sequence. (a) Pulse sequence for normal entanglement swapping. The sequence is divided into 3 successive steps: 1 . entangled state preparation; 2 . Bell state measurement $\left(Q_{2}\right.$ and $\left.Q_{3}\right)$; and 3. quantum state tomography $\left(Q_{1}\right.$ and $\left.Q_{4}\right)$. During the first step, each of the two qubit pairs $Q_{1}-Q_{2}$ and $Q_{3}-Q_{4}$ is tuned on resonance respectively for a specific time (about $153 \mathrm{~ns}$ for $Q_{1}$ and $Q_{2}$ and $114 \mathrm{~ns}$ for $Q_{3}$ and $Q_{4}$ ) with a rectangular pulse, realizing the $\sqrt{i \mathrm{SWAP}}$ gate. The $\pi$-rotation used in the first step and $\pi / 2$-rotation used in the third step are realized by microwave pulses with a length of $40 \mathrm{~ns}$ and a full-width half maximum of $20 \mathrm{~ns}$. The second step involves a dressed-state phase gate with a duration of about $219 \mathrm{~ns}$ and a multiplexed readout pulse of about $800 \mathrm{~ns}$, during which two weak resonant drives are applied to $Q_{1}$ and $Q_{4}$ respectively to minimize the dephasing effect (Dotted blue box). (b) Pulse sequence for delayed-choice entanglement swapping. Compared with (a), the temporal orders of the second $\left(Q_{2}-Q_{3}\right.$ Bell state measurement) and third steps $\left(Q_{1}-\right.$ $Q_{4}$ quantum state tomography) are reversed. Similarly, $Q_{2}$ and $Q_{3}$ are protected from dephasing noise during the readout of $Q_{1}$ and $Q_{4}$ (Dotted blue box). (c) Pulse sequence for delayed-choice separable-state projection. Here $Q_{2}$ and $Q_{3}$ are measured in the computational basis, which a posteriori projects $Q_{1}$ and $Q_{4}$ to a separable state.

$Q_{1}$ and $Q_{4}$ reconstructed from the four data subsets associated with the $Q_{2}-Q_{3}$ measurement outcomes $\left|0_{2}\right\rangle\left|0_{3}\right\rangle$, 
$\left|0_{2}\right\rangle\left|1_{3}\right\rangle,\left|1_{2}\right\rangle\left|0_{3}\right\rangle$, and $\left|1_{2}\right\rangle\left|1_{3}\right\rangle$ are displayed in Fig. 4(a)(d) of the main text, respectively. The pulse sequence with the choice of measurement in the computational basis is shown in Fig. S3(c), where the dressed-state phase gate is omitted before the detection of the states of $Q_{2}$ and $Q_{3}$, and $Q_{2}-Q_{3}$ readout pulse is applied about $40 \mathrm{~ns}$ after the end of $Q_{1}-Q_{4}$ readout pulse. In this case, the $Q_{1}-Q_{4}$ density matrices reconstructed from the four subsets associated with the $Q_{2}-Q_{3}$ measurement outcomes $\left|0_{2}\right\rangle\left|0_{3}\right\rangle,\left|0_{2}\right\rangle\left|1_{3}\right\rangle,\left|1_{2}\right\rangle\left|0_{3}\right\rangle$, and $\left|1_{2}\right\rangle\left|1_{3}\right\rangle$ are displayed in Fig. 4(e)-(h) of the main text, respectively.

As can be seen from Fig. S3(a), $Q_{2}-Q_{3}$ Bell state measurement is enabled by the combination of a dressedstate phase gate operation and multiplexed readout pulse which totally lasts about 1000 ns. During this period of time $Q_{1}$ and $Q_{4}$ are idled and thus endure decoherence effects. To mitigate these effects, following the dephasing suppression scheme proposed in Ref. [3], we apply weak continuous and resonant drives to these two qubits respectively (dotted blue box) during their idle time. As the phase is reversed in the middle of the driving pulse, the dephasing effect is reduced significantly, thus the quantum state of $Q_{1}$ and $Q_{4}$ can be well protected. The same method is employed in delayed-choice entanglement swapping experiment shown in Fig. S3(b), where the protection pulse is applied to $Q_{2}$ and $Q_{3}$.

In our experiment, $Q_{1}-Q_{4}$ joint density matrices associated with different $Q_{2}-Q_{3}$ measurement outcomes are reconstructed by postselection. For each of the three experiments shown in Fig. S3, we measure $2^{4}$ probabilities labelled as $P^{k}=\left\{P_{0_{1} 0_{2} 0_{3} 0_{4}}^{k}, P_{0_{1} 0_{2} 0_{3} 1_{4}}^{k}, P_{0_{1} 0_{2} 1_{3} 0_{4}}^{k}\right.$, ..., $\left.P_{1_{1} 1_{2} 1_{3} 1_{4}}^{k}\right\}$, where $k$ is the index of the $3^{2}$ tomographic operations applied to $Q_{1}$ and $Q_{4}$ before the joint readout. After readout correction, the probabilities are then sorted into four subsets, each of which is associated with one of $Q_{2}-Q_{3}$ measurement outcomes $\left\{\left|0_{2}\right\rangle\left|0_{3}\right\rangle\right.$, $\left.\left|0_{2}\right\rangle\left|1_{3}\right\rangle,\left|1_{2}\right\rangle\left|0_{3}\right\rangle,\left|1_{2}\right\rangle\left|1_{3}\right\rangle\right\}$. For example, probabilities $P_{0_{2} 0_{3}}^{k}=\left\{P_{0_{1} 0_{2} 0_{3} 0_{4}}^{k}, P_{0_{1} 0_{2} 0_{3} 1_{4}}^{k}, P_{1_{1} 0_{2} 0_{3} 0_{4}}^{k}, P_{1_{1} 0_{2} 0_{3} 1_{4}}^{k}\right\}$ are extracted to reconstruct the density matrix of $Q_{1}$ and $Q_{4}$ on condition that $Q_{2}$ and $Q_{3}$ are in $\left|0_{2}\right\rangle\left|0_{3}\right\rangle$ state. The same method applies to other cases.

\section{RESULTS WITHOUT READOUT ERROR CORRECTIONS FOR BELL STATE MEASUREMENT}

To better justify the deterministic feature of our experiment, we have also reconstructed the output density matrices without performing readout error corrections for the Bell state measurement, with the results for the normal entanglement swapping and delayed-choice entanglement swapping shown in Fig. S4 and S5, respectively. For the normal entanglement swapping, the fidelities of the reconstructed $Q_{1}-Q_{4}$ output density matrices associated with the $Q_{2}-Q_{3}$ Bell state measurement outcomes $\left\{\left|0_{2}\right\rangle\left|0_{3}\right\rangle,\left|0_{2}\right\rangle\left|1_{3}\right\rangle,\left|1_{2}\right\rangle\left|0_{3}\right\rangle,\left|1_{2}\right\rangle\left|1_{3}\right\rangle\right\}$ are $0.767 \pm 0.008$, $0.787 \pm 0.009,0.796 \pm 0.010$, and $0.839 \pm 0.010$, and the cor- responding concurrences are $0.540 \pm 0.017,0.590 \pm 0.016$, $0.603 \pm 0.022$, and $0.691 \pm 0.020$, respectively. For the delayed-choice entanglement swapping, the fidelities of the four conditional $Q_{1}-Q_{4}$ output density matrices are $0.749 \pm 0.009,0.778 \pm 0.010,0.803 \pm 0.009$, and $0.828 \pm 0.010$, while the corresponding concurrences are $0.522 \pm 0.019,0.584 \pm 0.020,0.615 \pm 0.018$, and $0.667 \pm 0.018$, respectively. These results unambiguously demonstrate that $Q_{1}$ and $Q_{4}$ are deterministically projected onto an entangled state after the swapping operation.
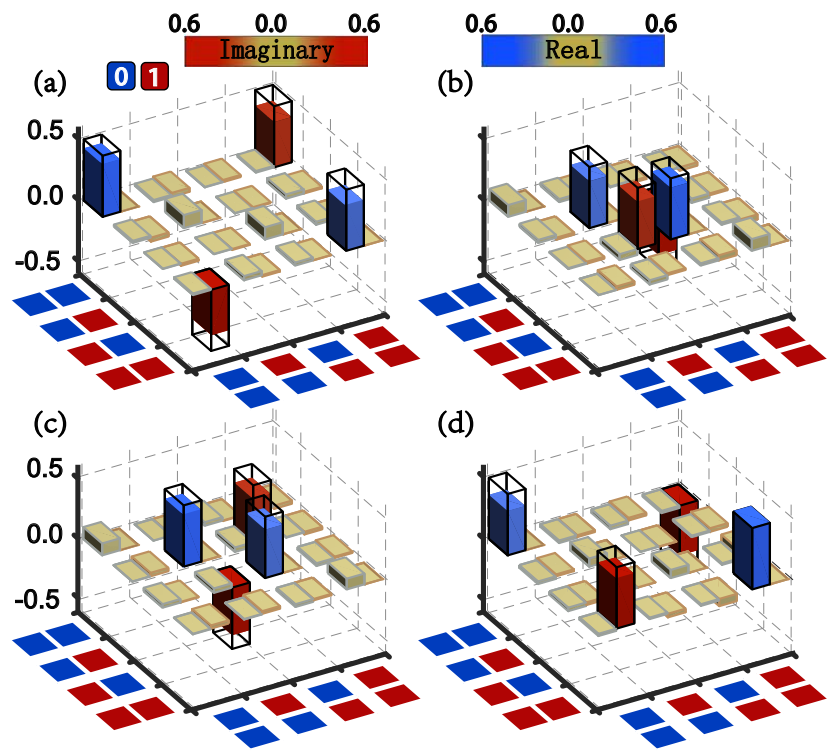

(d)

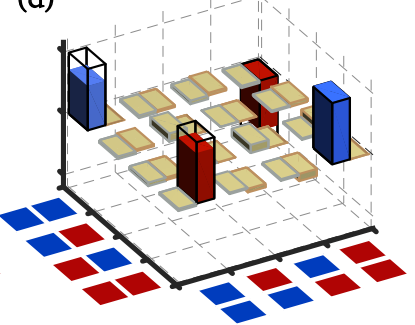

FIG. S4: Measured $Q_{1}-Q_{4}$ density matrices conditional on the four $Q_{2}-Q_{3}$ measurement outcomes: (a) $\left|0_{2}\right\rangle\left|0_{3}\right\rangle$; (b) $\left|0_{2}\right\rangle\left|1_{3}\right\rangle$; (c) $\left|1_{2}\right\rangle\left|0_{3}\right\rangle ;$ (d) $\left|1_{2}\right\rangle\left|1_{3}\right\rangle$. The results are obtained without performing readout error corrections for the Bell state measurement.

\section{NUMERICAL RESULTS}

The imperfections of the experimental results mainly come from the decoherence during the process. Numerical simulations with decoherence considered are performed to confirm the experimental outcomes. We use Lindblad master equation to model the state evolution under the Hamiltonian in section 1 , with $T_{1, j}$ measured in experiment. In simulation, we set the pure dephasing time of $Q_{j}$ to be $T_{\phi, j}^{D D}$ listed in Table S1 due to the fact that a resonant continuous drive is applied to each qubit to dynamically decouple it from dephasing noises when there is a significant interval between the Bell state preparation and its state readout [3]. We note that although no continuous drive is applied during the initial Bell state preparation, the dephasing is also greatly depressed due to the qubit-qubit interaction, which has been discussed elsewhere [4].

Numerical simulation shows the product of $Q_{1}-Q_{2}$ and $Q_{3}-Q_{4}$ Bell states produced by swapping interactions 


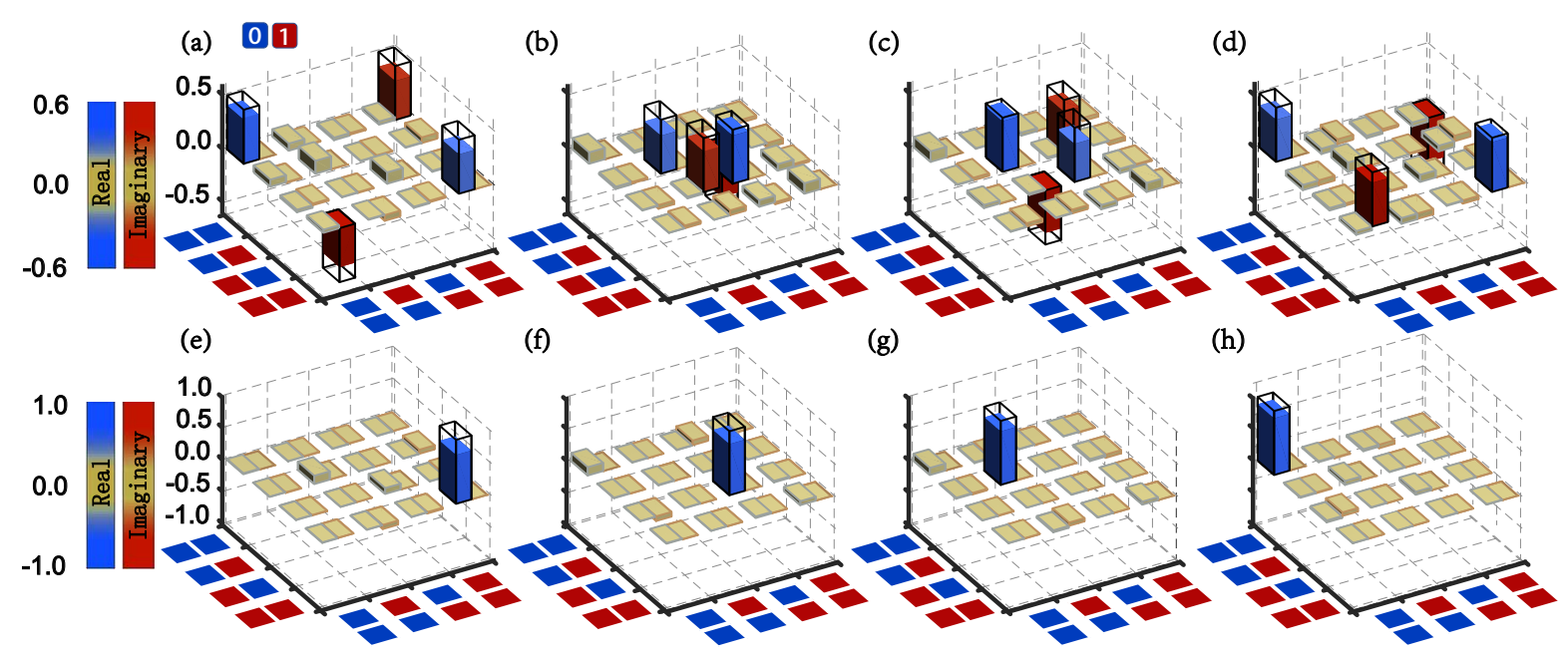

FIG. S5: Measured $Q_{1}-Q_{4}$ density matrices conditional on outcomes of delayed-choice $Q_{2}-Q_{3}$ measurement. (a)-(d) Results obtained from the four subsets of data correlated with the outcomes $\left\{\left|0_{2}\right\rangle\left|0_{3}\right\rangle,\left|0_{2}\right\rangle\left|1_{3}\right\rangle,\left|1_{2}\right\rangle\left|0_{3}\right\rangle,\left|1_{2}\right\rangle\left|1_{3}\right\rangle\right\}$ of $Q_{2}$ - $Q_{3}$ measurement performed after the dressed-state phase gate. (e)-(h) Results obtained from the four subsets of data correlated with the outcomes of the later $Q_{2}-Q_{3}$ measurement without the dressed-state phase gate. All these results are obtained without performing readout error corrections for the Bell state measurement.

has a fidelity of 0.979 with respect to the ideal state, which is in well agreement with the measured value of $0.971 \pm 0.009$. For the dressed-state phase gate, the numerical results yield a fidelity of 0.975 , slightly higher than the experimental value $0.966 \pm 0.005$.

The measured probabilities of different $Q_{2}-Q_{3}$ basis states, and the associated $Q_{1}-Q_{4}$ output state fidelities and concurrences, together with the simulated results, for the normal entanglement swapping, delayed-choice entanglement swapping, and delayed-choice separablestate projection are shown in Tables S2, S3, and S4, respectively. The experimental results are in good agreement with the numerical simulation overall. Fidelities in delayed-choice entanglement swapping are higher than those in the non-delayed case due to the fact that $Q_{1}$ $Q_{4}$ joint state is detected earlier so that the measured data is less affected by decoherence effects. The slight differences between the experimental and numerical results are partly due to the fact that $T_{\phi, j}^{D D}$, which is taken as the effective dephasing time of $Q_{j}$ in our simulation, does not perfectly characterize the effect of the dephasing noises under continuous driving. As demonstrated in Ref. [3], the qubit's dephasing time depends not only on intensity of the applied drive but also on its effective interaction strengths with the others, which is not taken into account in the simulation. Another reason for these differences is imperfect control of the parameters of the continuous drives, whose fluctuations affect the performance of the dressed-phase gate and introduce an extra error to the protected qubits.

According to the numerical simulation, after the parallel Bell state preparations $Q_{1}-Q_{4}$ has a small probability of being populated in $\left|0_{1} 1_{4}\right\rangle$ when $Q_{2}-Q_{3}$ is projected to $\left|0_{2} 1_{3}\right\rangle$ due to the imperfect large detuning conditions, so that the elements associated with $\left|0_{1} 1_{4}\right\rangle\left\langle 1_{1} 0_{4}\right|$ and $\left|1_{1} 0_{4}\right\rangle\left\langle 0_{1} 1_{4}\right|$ of the corresponding projected $Q_{1}-Q_{4}$ density matrix have a magnitude of about 0.065 , which accounts for the calculated $Q_{1}-Q_{4}$ concurrence (0.069) corresponding to the $Q_{2}-Q_{3}$ output $\left|0_{2} 1_{3}\right\rangle$ under the delayed-choice separable-state projection. However, in experiment the measured magnitude of these off-diagonal elements is only about 0.02 due to imperfect timing, to which these elements are extremely sensitive because they oscillate very fast during the parallel entangling gates. This magnitude is further reduced to about 0.007 by the delay of projection, during which extra noises are introduced. Consequently the corresponding concurrence is too small to detect in experiment. The difference between the calculated $Q_{1}-Q_{4}$ concurrence and the measured result corresponding to the $Q_{2}-Q_{3}$ output $\left|1_{2} 0_{3}\right\rangle$, shown in Table S4, is due to the same reason.

\section{EXPERIMENTAL SETUP}

Fig. S6 shows the whole control and readout layout. The qubit control and readout are implemented by FPGA-based digital-to-analog converter (DAC) and analog-to-digital converter (ADC). Each control signal output, measurement signal input and output channel is filtered by a custom Gaussian low-pass filter with the bandwidth of $7.5 \mathrm{GHz}$ (for qubit XY control and for readin and readout control) and of $500 \mathrm{MHz}$ (for qubit $\mathrm{Z}$ control), respectively. The XY control signal for each qubit comes from the mixing of the low-frequency signals sent by two independent DAC channels I/Q and the high-frequency signal produced by the microwave source, which realizes the fast qubit flipping at the nanosecond scale. While the qubit $\mathrm{Z}$ control signal is directly sent from DAC without mixing, which enables the fast fre- 


\begin{tabular}{c|c|c}
\hline \hline $\begin{array}{c}Q_{2}-Q_{3} \text { probability distribution } \\
\text { Experiment(Simulation) }\end{array}$ & $\begin{array}{c}Q_{1-Q_{4}} \text { Fidelity } \\
\text { Experiment (Simulation) }\end{array}$ & $\begin{array}{c}Q_{1}-Q_{4} \text { Concurrence } \\
\text { Experiment (Simulation) }\end{array}$ \\
\hline$\left|0_{2}\right\rangle\left|0_{3}\right\rangle: 0.249 \pm 0.004(0.250)$ & $\left|\Phi_{1,4}^{+}\right\rangle: 0.893 \pm 0.010(0.895)$ & $0.794 \pm 0.020(0.794)$ \\
$\left|0_{2}\right\rangle\left|1_{3}\right\rangle: 0.250 \pm 0.006(0.257)$ & $\left|\Psi_{1,4}^{-}\right\rangle: 0.879 \pm 0.010(0.895)$ & $0.779 \pm 0.020(0.792)$ \\
$\left|1_{2}\right\rangle\left|0_{3}\right\rangle: 0.255 \pm 0.005(0.246)$ & $\left|\Psi_{1,4}^{+}\right\rangle: 0.872 \pm 0.011(0.885)$ & $0.758 \pm 0.024(0.781)$ \\
$\left|1_{2}\right\rangle\left|1_{3}\right\rangle: 0.246 \pm 0.004(0.247)$ & $\left|\Phi_{1,4}^{-}\right\rangle: 0.884 \pm 0.010(0.893)$ & $0.785 \pm 0.021(0.792)$ \\
\hline \hline
\end{tabular}

TABLE S2: Measured and calculated probabilities of different $Q_{2}-Q_{3}$ basis states, and the associated $Q_{1}-Q_{4}$ output state fidelities and concurrences for the normal entanglement swapping.

\begin{tabular}{c|c|c}
\hline \hline $\begin{array}{c}Q_{2}-Q_{3} \text { probability distribution } \\
\text { Experiment(Simulation) }\end{array}$ & $\begin{array}{c}Q_{1-Q_{4}} \text { Fidelity } \\
\text { Experiment (Simulation) }\end{array}$ & $\begin{array}{c}Q_{1}-Q_{4} \text { Concurrence } \\
\text { Experiment (Simulation) }\end{array}$ \\
\hline$\left|0_{2}\right\rangle\left|0_{3}\right\rangle: 0.248 \pm 0.004(0.251)$ & $\left|\Phi_{1,4}^{+}\right\rangle: 0.891 \pm 0.012(0.908)$ & $0.815 \pm 0.026(0.820)$ \\
$\left|0_{2}\right\rangle\left|1_{3}\right\rangle: 0.256 \pm 0.005(0.258)$ & $\left|\Psi_{1,4}^{-}\right\rangle: 0.891 \pm 0.012(0.913)$ & $0.816 \pm 0.024(0.827)$ \\
$\left|1_{2}\right\rangle\left|0_{3}\right\rangle: 0.248 \pm 0.006(0.243)$ & $\left|\Psi_{1,4}^{+}\right\rangle: 0.896 \pm 0.010(0.899)$ & $0.806 \pm 0.022(0.808)$ \\
$\left|1_{2}\right\rangle\left|1_{3}\right\rangle: 0.248 \pm 0.006(0.248)$ & $\left|\Phi_{1,4}^{-}\right\rangle: 0.897 \pm 0.010(0.908)$ & $0.807 \pm 0.019(0.821)$ \\
\hline \hline
\end{tabular}

TABLE S3: $\quad$ Measured and calculated probabilities of different $Q_{2}-Q_{3}$ basis states, and the associated $Q_{1}-Q_{4}$ output state fidelities and concurrences for the delayed-choice entanglement swapping.

\begin{tabular}{c|c|c}
\hline \hline $\begin{array}{c}Q_{2}-Q_{3} \text { probability distribution } \\
\text { Experiment(Simulation) }\end{array}$ & $\begin{array}{c}Q_{1}-Q_{4} \text { Fidelity } \\
\text { Experiment (Simulation) }\end{array}$ & $\begin{array}{c}Q_{1}-Q_{4} \text { Concurrence } \\
\text { Experiment (Simulation) }\end{array}$ \\
\hline$\left|0_{2}\right\rangle\left|0_{3}\right\rangle: 0.258 \pm 0.005(0.258)$ & $\left|1_{1}\right\rangle\left|1_{4}\right\rangle: 0.907 \pm 0.011(0.932)$ & $0.016 \pm 0.018(0.000)$ \\
$\left|0_{2}\right\rangle\left|1_{3}\right\rangle: 0.250 \pm 0.005(0.257)$ & $\left|1_{1}\right\rangle\left|0_{4}\right\rangle: 0.914 \pm 0.009(0.934)$ & $0.005 \pm 0.007(0.069)$ \\
$\left|1_{2}\right\rangle\left|0_{3}\right\rangle: 0.251 \pm 0.005(0.245)$ & $\left|0_{1}\right\rangle\left|1_{4}\right\rangle: 0.930 \pm 0.009(0.946)$ & $0.004 \pm 0.005(0.038)$ \\
$\left|1_{2}\right\rangle\left|1_{3}\right\rangle: 0.241 \pm 0.008(0.240)$ & $\left|0_{1}\right\rangle\left|0_{4}\right\rangle: 0.949 \pm 0.008(0.958)$ & $0.015 \pm 0.011(0.000)$ \\
\hline \hline
\end{tabular}

TABLE S4: Measured and calculated probabilities of different $Q_{2}-Q_{3}$ basis states, and the associated $Q_{1}-Q_{4}$ output state fidelities and concurrences for the delayed-choice separable-state projection.

quency tuning. The frequency can also be tuned at a slow rate through a direct-current (DC) biasing line, where the signal generated from a low-frequency electrical source is filtered by a RC filter and a $80 \mathrm{MHz}$ low-pass filter before it is converged to qubit $\mathrm{Z}$ control line through a bias tee at the low temperature stage.

The output signal from the readout feed line is amplified sequentially by the impedance-transformed Josephson parametric amplifier (JPA), high electron mobility transistor (HEMT) and room temperature amplifiers before it is captured and demodulated by ADC. Four cryogenic unidirectional circulators with low insertion loss are added between JPA and the device to block the reflections and noise emitted from the input of the HEMT at the $4 \mathrm{~K}$ stage. The JPA is pumped by an independent microwave signal source through the pumping line, where the signal is filtered by a $13.1 \mathrm{GHz}$ bandpass filter; the JPA's amplification band is tunable with a DC biasing line, the signal on which is filtered with a RC filter as well as a low-pass filter before it is converged to a bias tee that connects the JPA pump line.

Every control line is balanced with some attenuators at different temperature stage in the dilution refrigerator to prevent the unwanted noises from affecting the device.

\section{QUBIT READOUT}

To realize the single-shot readout demanded in this scheme, each $Q_{j}$ is capacitively coupled to its own readout resonator $R_{j}$, which can be probed by microwave pulses output from the room-temperature DAC. During the measurement, the readout pulse carrying 4 tones resonant with each $R_{j}$ respectively are applied through a common transmission line coupled to all $R_{j}$ s. As the resonators are pumped with photons which dispersively interact with the qubits, the state information is encoded in the readout signal returned from the device. At the end of the microwave driving, the photons accumulated in the resonator will leak into the transmission line with a decay rate of $\kappa_{j}^{r}$ listed in Table S1. The returned signal is amplified by a JPA (see supplemental materials of reference [2]) to enhance the signal-to-noise ratio before it is captured and further demodulated with uniform integration weight by a room-temperature ADC. The $I$ and $Q$ values extracted from the demodulation process with each qubit in ground and excited state are illustrated in Fig. S7(b)-(e). The results are obtained from 3000 repetitive measurements. Fig. S7(a) shows the signal transmission spectra with JPA switched on and off. The read pulse for each qubit has the length of about $0.8 \mu s$ or $1.1 \mu s$, which depends on the temporal order of measurement, as can be seen from Fig. S3. 


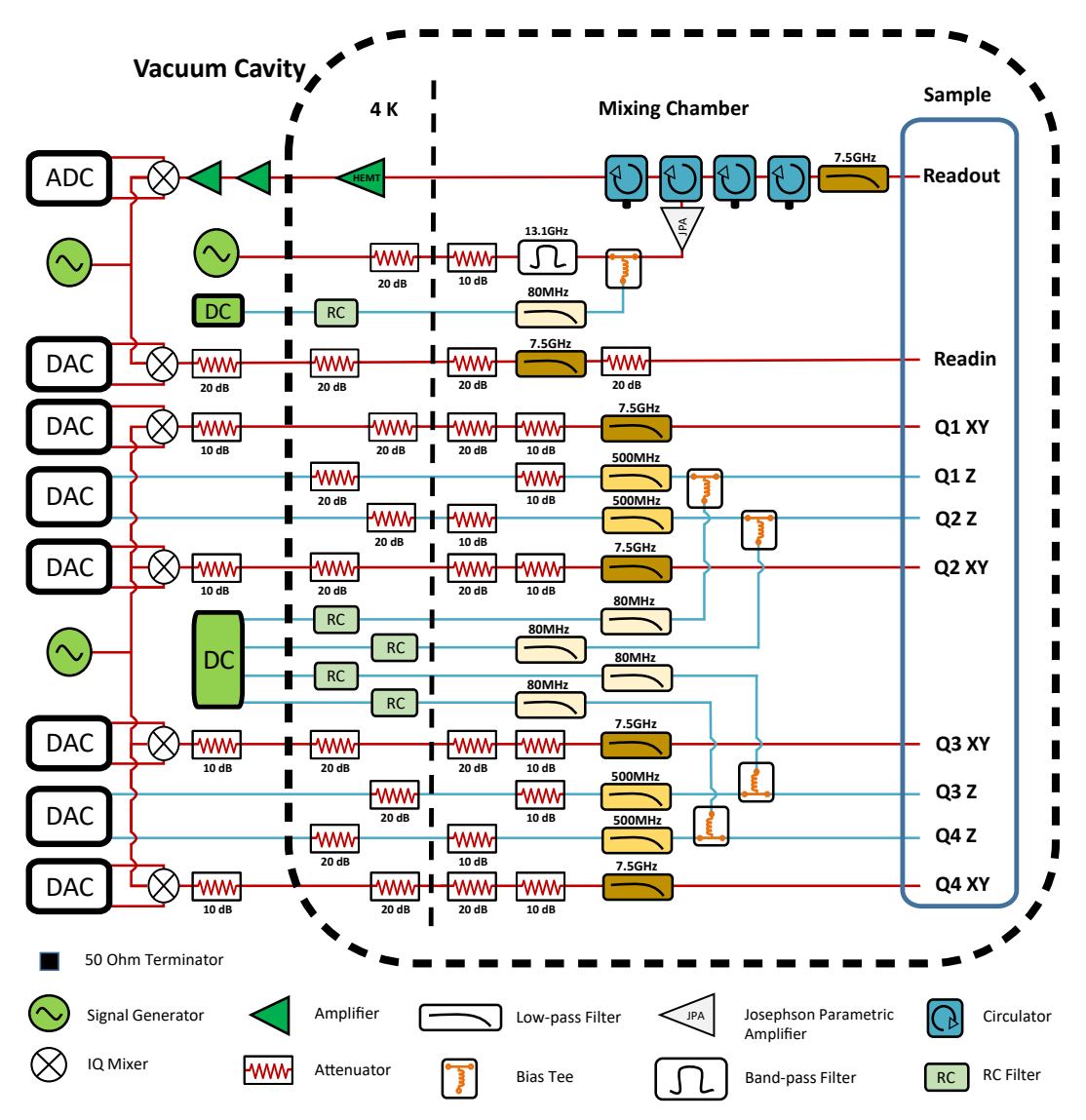

FIG. S6: The layout of the experimental setup. Diagram showing all the control and wiring for the experimental setup. The electronics for the qubit control, JPA control, and qubit readout are shown from the bottom to the top. The red line corresponds to the qubit XY control and measurement, while the blue line corresponds to the qubit $\mathrm{Z}$ control. The qubit XY control signal is from the mixing of the signals of the two DAC channels I/Q and the microwave source, while the qubit $\mathrm{Z}$ control is directly produced by DAC without mixing. These two types of qubit control realize the fast qubit flippling and frequency tuning at the nanosecond scale, respectively. Each qubit also possesses a direct-current biasing line converging to the $\mathrm{Z}$ line for the larger-scale frequency modulation but at a slow rate. The output signal is amplified sequentially by JPA, HEMT, room temperature amplifiers, and finally captured and demodulated by ADC. The JPA is pumped by an independent microwave signal source and its amplification band is tunable with a DC bias. Each control line goes through same attenuators and filters at each temperature stage in the dilution refrigerator to block the unwanted noises out the device.

The measurment effeciencies are also charaterized with the method used in Ref. [5]. By comparing the results of two kinds of experiments: the photon-induced dephasing measured by Ramsey interference and the signal-tonoise ratio measurement, we extract the measurement efficiency, which has the values of $16 \mathrm{~dB}, 13 \mathrm{~dB}, 16 \mathrm{~dB}$ and $15 \mathrm{~dB}$ for $Q_{1}, Q_{2}, Q_{3}$ and $Q_{4}$, respectively. Fig. S8 shows the results of measurement efficiency by taking $Q_{2}$ as an example.
[1] C. Song, S.-B. Zheng, P. Zhang, K. Xu, L. Zhang, Q. Guo, W. Liu, D. Xu, H. Deng, K. Huang, D. Zheng, X. Zhu, and H. Wang, Nat. Commun. 8, 1061 (2017).

[2] C. Song, K. Xu, W. Liu, C.-P. Yang, S.-B. Zheng, H. Deng, Q. Xie, K. Huang, Q. Guo, L. Zhang, P. Zhang, D. Xu, D. Zheng, X. Zhu, H. Wang, Y.-A. Chen, C.-Y. Lu, S. Han, and J.-W. Pan, Phys. Rev. Lett. 119, 180511 (2017).

[3] Q. Guo, S.-B. Zheng, J. Wang, C. Song, P. Zhang, K. Li, W. Liu, H. Deng, K. Huang, D. Zheng, X. Zhu, H. Wang,
C.-Y. Lu, and J.-W. Pan, Phys. Rev. Lett. 121, 130501 (2018).

[4] K. Xu, J.-J. Chen, Y. Zeng, Y.-R. Zhang, C. Song, W. Liu, Q. Guo, P. Zhang, D. Xu, H. Deng, K. Huang, H. Wang, X. Zhu, D. Zheng, and H. Fan, Phys. Rev. Lett. 120, 050507 (2018).

[5] D. Sank, Ph. D. thesis, Univerisity of California Santa Barbara, 2014. 

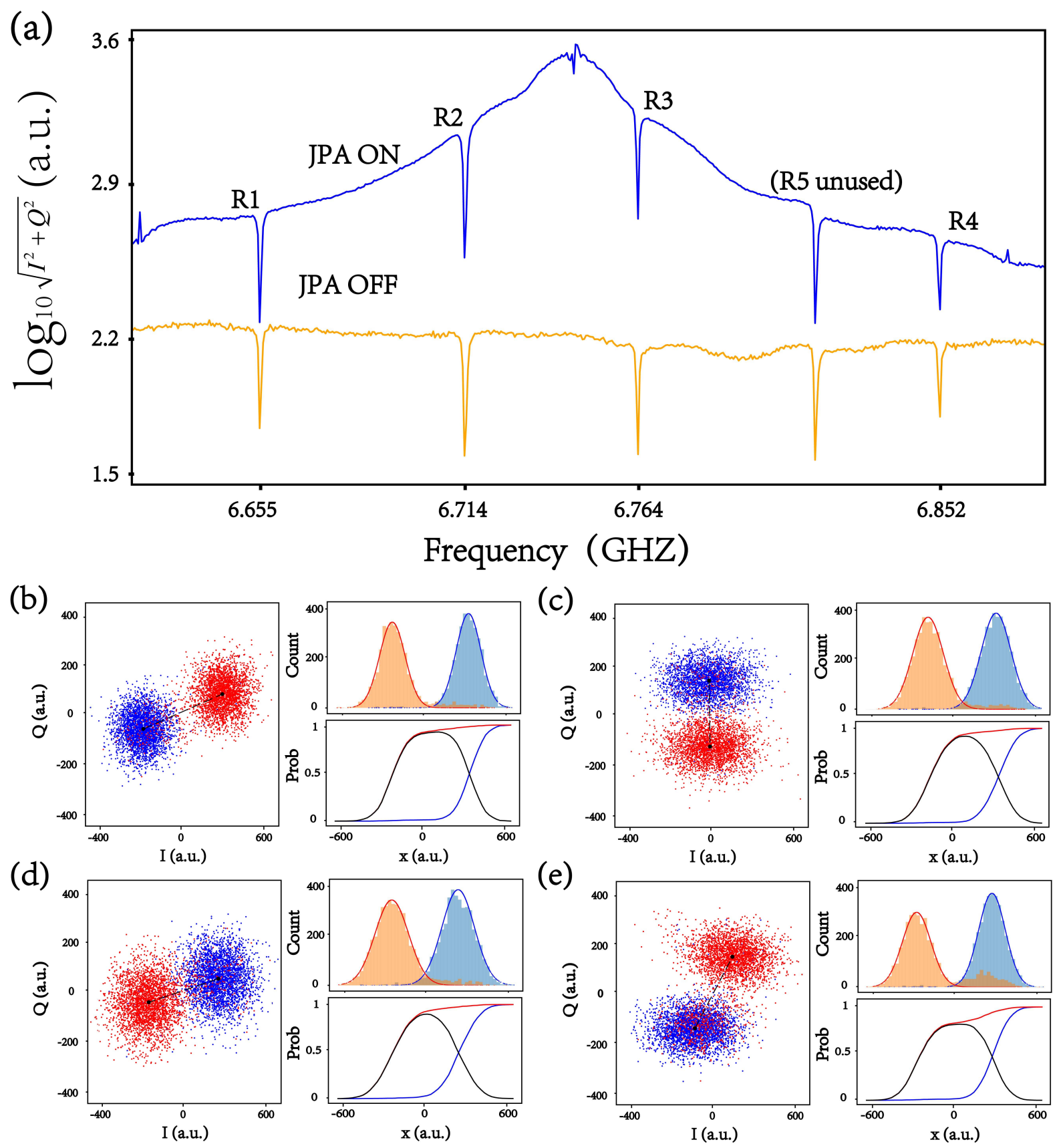

FIG. S7: (a) Signal transmission spectra through the measurement circuit (all qubits are in $|0\rangle$ ). The amplitudes of the demodulated signal are plotted as functions of the signal frequency, the two curves correspond to the cases when JPA is "ON" (blue) and "OFF" (orange), respectively. Five transmission dips appear at the readout resonators, which are labeled from $R_{1}$ to $R_{5}$ ( $R_{5}$ is unused). The increased values in the longitudinal direction of the blue curve as compared to the orange one manifests the JPA's ability to amplify the signals. The measured gain ranges from $8 \mathrm{dBm}$ to $22 \mathrm{dBm}$. (b) $Q_{1}$, (c) $Q_{2}$, (d) $Q_{3}$, (e) $Q_{4}$. Left panel: Demodulated I-Q data for single-shot qubit state differentiation, where blue (red) dots are measured I-Q values when qubit is prepared in $|0\rangle(|1\rangle)$. The results are achieved through 3000 repetitive measurements. Top-right panel: Distribution histogram along the axis (depicted as the dotted line) in the left panel. Bottom-right panel: Measurement visibility by integrating the histogram along the dotted line axis, which has the value of about $90 \%, 90 \%, 88 \%$, and $80 \%$ for $Q_{1}, Q_{2}, Q_{3}$, and $Q_{4}$, respectively. 


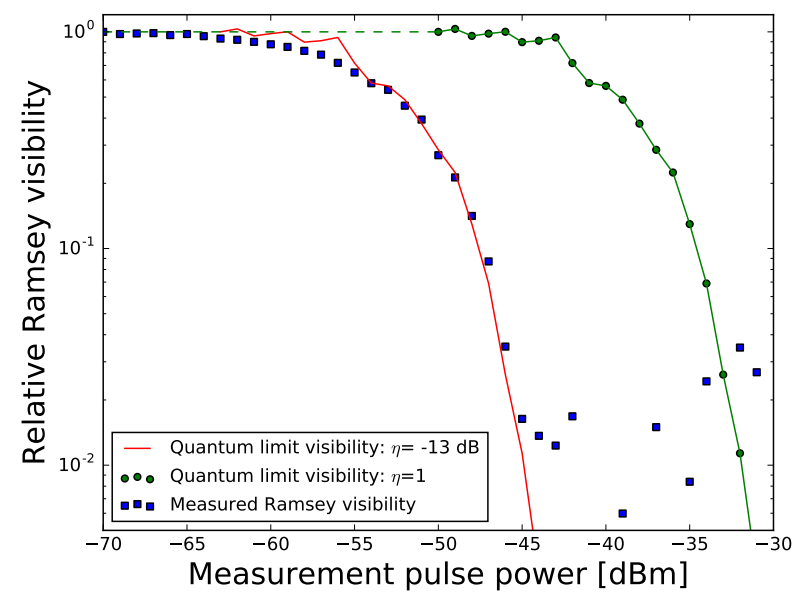

FIG. S8: Measurement efficiency of the system $\left(Q_{2}\right.$ as an example). Two kinds of experiments are performed. One is the measurement of the photon induced qubit dephasing which can be charaterized by Ramsey fringe sequence, where a measurement pulse with a variable power is applied in between the two $\pi / 2$-pulses, the relative fringe visibility is measured and plotted as a function of measurement pulse power (shown as blue squares). This measurement reduces the visibility of the Ramsey fringes as the applied pulse dephases the qubit. Another is the measurement of the readout distinguishability between the qubit's ground and excited state. This can be translated to a quantum limit on Ramsey fringe visibility via the relation: $\left|\rho_{10}\right| \leq \frac{1}{2} \exp \left(-\frac{S N R}{4}\right)$ (green circles), where $\rho_{10}$ is the off-diagonal term of the density matrix of the state of the two-level system that indicates the phase coherence and SNR indicates the signal to noise ratio. The plot of this quantum limit is reshown by a left shift of it by $13 \mathrm{~dB}$ (red line), that passes through the points of the relative Ramsey fringe visibility. 